\title{
26016 - TRICUSPID ANNULAR VELOCITIES IN ATRIAL FIBRILLATION
}

\section{Claude Tousignant MD, Richard Bowry, MB BS; Andrew Miller, MB BS, St. Michael's Hospital, Toronto, ONTARIO, Canada}

INTRODUCTION: Tricuspid annular motion has been used to assess right ventricular (RV) systolic function(1). The aim of this study was to investigate the effect of heart rate (HR) on the load independent isovolemic acceleration (IVA) and load dependent systolic descent velocity (S wave)(2). Patients with atrial fibrillation (AF) were used. METHODS: After Institutional Review Board approval and informed consent, 22 post cardiac surgery patients with AF were enrolled. Using a GE vivid 7 with $1.7 / 3.4 \mathrm{MHz}$ probe, an apical four chamber view was obtained. A tissue Doppler color sector was applied to the lateral tricuspid annulus. 8 loops of at least 20 cycles with a frame rate $>200$ fps were recorded and stored digitally. Using echopac quantitative analysis software a $6 \times 6 \mathrm{~mm}$ sample was placed on the ventricular side of the tricuspid annulus and adjusted to maximize the $\mathrm{S}$ wave velocity. For each beat, IVA $(\mathrm{m} / \mathrm{s} 2)$ and peak $\mathrm{S}$ wave velocity $(\mathrm{cm} / \mathrm{s})$ were measured (figure $1 \mathrm{~A})$ and the corresponding heart rate $(\mathrm{HR})$ was calculated from the preceding R-R interval. At least 40 beats were measured per patient. Best-fit regression was used to assess the relationship between HR and both IVA and S wave velocity. The measurements were divided into HR groups (figure 1B) and analyzed using Kruskal-Wallis one way analysis of variance on ranks.

RESULTS: 7 patients were excluded from analysis, there were 12 males and 3 females, average age: $66 \pm 12$ years. In all, 715 beats were analyzed. There was no difference in IVA between groups 1 and 2 . In groups 3 to 5 IVA increased significantly when compared to each previous HR group $(\mathrm{p}<0.05)$ (figure 1B). There was no significant difference in $S$ wave velocity in groups 1 to 3 . The $S$ wave velocity was significantly lower in groups 4 and 5 when compared to groups 1 and 2. $(\mathrm{p}<0.05)$ (figure1B). For all patients, there was a positive linear correlation between IVA and HR $(r=0.49, p<0.001)$. For $\mathrm{S}$ wave and $\mathrm{HR}$, best fit was achieved using a second order polynomial equation $(\mathrm{r}=0.50, \mathrm{p}<0.001)$.

DISCUSSION: As the HR increased, there was a significant rise in the IVA when HR was above $100 \mathrm{bpm}$ and a significant reduction in the $\mathrm{S}$ wave with HR greater than 120 bpm. The observed increases in IVA were likely the result of the Bowditch effect(3). For the $\mathrm{S}$ wave, the decrease was likely due to limitations in preload due to HR. Indeed the $S$ wave may have fallen faster in patients with lower volume status. When all the data were pooled, there was only a modest correlation for IVA and S wave when compared to $\mathrm{HR}$, possibly the result of variable RV systolic dysfunction and preload. Tricuspid annular motion may be useful in assessing RV systolic function however, when HR exceeds $100 \mathrm{bpm}$ the increase in IVA needs to be taken in consideration. HR-induced preload limitations were likely the cause of the decrease in $\mathrm{S}$ wave.

REFERENCES:

1. Am Heart J 2000 139: 710-15

2. Circulation 2002 5: 1693-99

3. Arb Physiol Aust Leipzig 1871 6: 139-176 


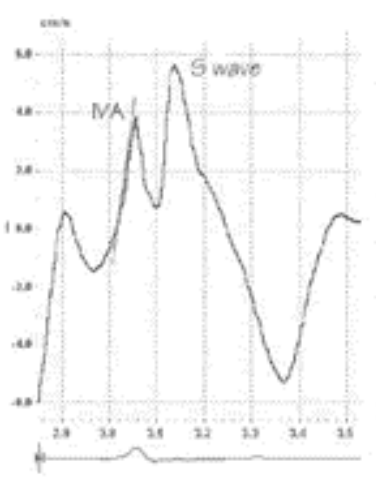

Tricuspid Annular Velocites

A) Trisuepis ancilar velixity trace ning

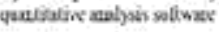

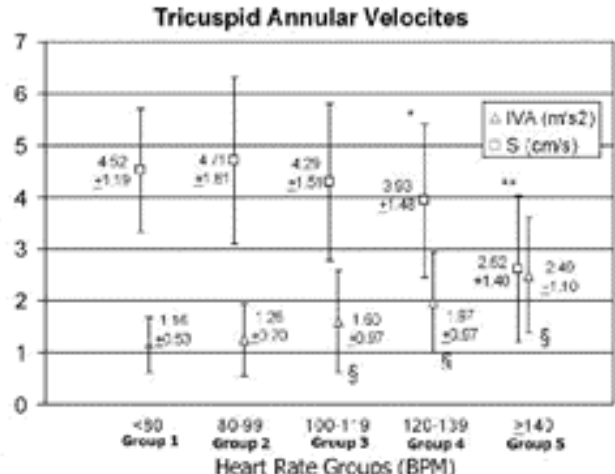

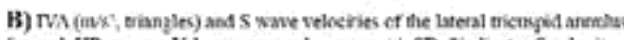

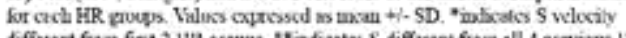

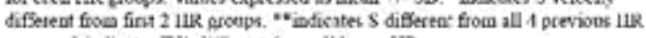
gruega. $\xi$ imbicales V. diflerest from all lower IIR goups. 